\title{
Deutschsprachige Artikel zur slawistischen Sprachwissenschaft als Mittel der fachlichen und sprachlichen Sozialisation - eine problematische Annäherung an die Disziplin
}

\author{
Ursula Doleschal und Anzhelika Scherling (Klagenfurt am Wörthersee)
}

\begin{abstract}
Research articles are studied by several branches of Applied Linguistics, such as text linguistics, stylistics, foreign language teaching or contrastive rhetoric. However, articles written in German have been scarcely investigated so far. In this contribution we study German articles in Slavic linguistics with regard to their use for the socialization of students into academia. We investigate the presence of the following contents as well as their position of occurrence in the texts: research question, methods and theoretical background. Thus we evaluate, if such articles are an adequate means to introduce students to the way the results of research are brought about. Our study shows that research questions are usually explicitly stated, while methods and theoretical background may remain implicit. It seems that the degree of implicitness relates to the degree of "traditionality" of the research involved, i. e., if the article is, e. g., a philological or a sociolinguistic one.
\end{abstract}

\section{$1 \quad$ Einleitung 1}

Der wissenschaftliche Artikel, insbesondere der "Research article", ist ein zentrales Thema der Forschung über wissenschaftliche Texte. Die Erforschung dieser Textsorte wird vor allem im Rahmen der kontrastiven Rhetorik betrieben, und so beschäftigen sich die meisten Arbeiten (wie bereits Pöckl 1995 feststellt) mit dem Kontrast zwischen Englisch und einer zweiten Sprache. Dabei werden die Merkmale des englischen Artikels oft explizit oder implizit zur Norm erhoben.

Deutschsprachige wissenschaftliche Artikel wurden bisher wenig erforscht. Als grundlegend sind die Arbeiten von Michael Clyne (1987, 1991) und Gabriele Graefen (1997) zu nennen. Mit der Arbeit von Mikaela Petkova-Kessanlis (2009) liegt eine Monografie über zwei Teiltexte des wissenschaftlichen Artikels vor: Einleitung und Zusammenfassung. Auf Grund dieser spärlichen Datenlage ist die Untersuchung wissenschaftlicher Artikel in deutscher Sprache ein dringendes Forschungsdesiderat.

An dieser Stelle geht es um eine Annäherung an eine spezielle Ausprägung der Textsorte

\footnotetext{
${ }^{1}$ Wir danken Sonja Kuri und unserem anonymen Rezensenten bzw. Rezensentin für wertvolle Hinweise zu einer früheren Version dieses Artikels und Gerhild Gram für Hilfe im letzten Moment.
}

Linguistik online 76, 2/16 - http://dx.doi.org/10.13092/lo.76.2815

CC by 3.0 
„wissenschaftlicher Artikel“ - um sprachwissenschaftliche Artikel aus dem Bereich der Slawistik. Deutschsprachige wissenschaftliche Literatur ist aber nicht nur ein Kommunikationsmittel innerhalb der slawistischen Forschungsgemeinschaft, sondern auch ein wichtiges Instrument der Sozialisation für Studierende der Slawistik, da diese in den ersten Studienjahren die Forschungsliteratur noch nicht in der slawischen Originalsprache lesen können. Auch daher gebührt den deutschsprachigen Artikeln besondere Aufmerksamkeit.

Im vorliegenden Beitrag stellen wir eine explorative Studie deutschsprachiger sprachwissenschaftlicher Artikel aus dem Bereich der Slawistik vor und gehen dabei sowohl auf soziolinguistische als auch didaktische Dimensionen des Themas ein. Die untersuchten Artikel zeigen relativ hohe textuelle Implizitheit und Indirektheit (vgl. Doleschal 2007). Warum das so ist diskutieren wir nach der Darstellung der Hintergründe und der Untersuchung selbst. Unsere Arbeit reiht sich in die Forschung zum „Deutschen als Wissenschaftssprache“ (Ammon 1998) ein.

\section{$2 \quad$ Forschungsdesign}

\subsection{Theoretischer Hintergrund und Hypothesen}

Unsere Forschung fußt auf einer didaktischen Maßnahme im Rahmen einer Lehrveranstaltung des Slawistikstudiums an der Universität Klagenfurt. Das sprachwissenschaftliche Proseminar ist die erste Lehrveranstaltung, in der sich die Studierenden selbstständig mit einem sprachwissenschaftlichen Thema auseinandersetzen und darüber ein Referat halten sowie eine Proseminararbeit im Umfang von 3000 Wörtern schreiben müssen. Dieses Proseminar soll im zweiten Studienjahr absolviert werden. Da die meisten Studierenden das Slawistikstudium ohne Vorkenntnisse in einer slawischen Sprache beginnen, sind sie zu diesem Zeitpunkt auf Grund ihrer Sprachkenntnisse (zumeist Niveau A2 des Gemeinsamen Europäischen Referenzrahmens) nicht in der Lage, sprachwissenschaftliche Literatur in der studierten Sprache (zur Wahl stehen Bosnisch, Kroatisch, Russisch, Serbisch, Slowenisch) zu rezipieren. Auch das Referat und die Proseminararbeit verfassen sie daher nicht in einer slawischen Sprache, sondern auf Deutsch. Das bedeutet, sie müssen hier sowohl die allgemeine deutsche Wissenschaftssprache als auch die fachspezifische Terminologie anwenden und brauchen dafür Vorbilder (vgl. Graefen 1997: 63-65, 93). Nach Graefen und Thielmann (2007: 67) sind im Verständnis der deutschsprachigen akademischen Sozialisation wissenschaftliche Artikel „Vorbild für studentische Arbeiten, mit denen die erfolgreiche Anpassung an wissenschaftliches Arbeiten bewiesen werden kann bzw. muss". Allein aus diesen didaktischen Gründen ist deutschsprachige wissenschaftliche Literatur für die deutschsprachige Slawistik sehr wichtig und kann nicht durch englischsprachige Texte ersetzt werden. $\mathrm{Zu}$ ergänzen ist zudem, dass die Lehre zumindest im Grundstudium zur Gänze in deutscher Sprache gehalten wird.

Die deutsche Sprache nimmt in der internationalen Slawistik traditionell eine wichtige Position ein, die auf die frühe Gründung slawistischer Lehrstühle an deutschsprachigen Universitäten (ab den 1840er Jahren, vgl. Zeil 1994: 150-195) und die führende Rolle der dortigen Sprachwissenschaftler, insbesondere der Junggrammatiker, zurückgeht. Deutsch ist 
bis heute eine der offiziellen Sprachen des Internationalen Slawistenkongresses (neben Englisch, Französisch und allen slawischen Sprachen). Dennoch ist Deutsch mittlerweile auch in der Slawistik eine nachgeordnete Sprache, die vor allem von nichtslawischen und allgemeinen SlawistInnen beherrscht wird. Wenn man von einem Publikum rezipiert werden will, das die jeweils untersuchte Sprache versteht, empfiehlt es sich, in jener zu publizieren, also z.B. auf Russisch bei einer russistischen Publikation oder auf Slowenisch bei einer slowenistischen. In manchen Schulen wie der Generativen Grammatik greift außerdem das Englische Platz.

In der deutschsprachigen Slawistik gibt es sechs (international) renommierte Fachzeitschriften, ${ }^{2}$ von denen drei im Arts and Humanities Citation Index gelistet sind. Diese Zeitschriften publizieren sowohl auf Deutsch als auch (seltener) in anderen Sprachen. Die Reviewpraxis ist nicht durchsichtig und wird wahrscheinlich von Fall zu Fall unterschiedlich gehandhabt. Außerdem gibt es eine Reihe von Verlagen, die slawistische Monografien und Sammelbände in deutscher Sprache in speziellen Reihen publizieren. Diese Zahl an Publikationsorganen setzt eine gewisse Größe der Diskursgemeinschaft (Swales 1990: 21-32) voraus, und tatsächlich gibt es allein in Deutschland etwa 100 Lehrstühle an 35 Instituten (www.slavistenverband.de/Verband.html, [22.12.2015]), in Österreich sind es sechs Institute mit etwa 15 Lehrstühlen (www.slawistik.at/institute, [22.12.2015]), in der deutschsprachigen Schweiz ebenfalls sechs Institute (www.sagw.ch/sags/die-gesellschaft/institute.html, [22.12.2015]). Andererseits ist diese Diskursgemeinschaft in Untergruppen, wie jene der slawistischen SprachwissenschaftlerInnen, untergliedert, die gut überschaubar sind, „man kennt sich“ also. Außerdem gibt es Gruppen von SprachwissenschaftlerInnen, die sich alljährlich zu Symposien treffen und ihre Beiträge in Sammelbänden oder einer der genannten Zeitschriften publizieren (z. B. Konstanzer Kreis, JungslawistInnen), ohne jedoch Forschungsgemeinschaften zu bilden. Das heißt, diese Gruppen werden nicht durch ein gemeinsames Thema, Forschungsprojekt oder eine gemeinsame Schule vereint.

Die Publikation von Forschungsartikeln (research articles) in Zeitschriften ist in der (internationalen) Slawistik bislang nicht von hervorragender Bedeutung - Monografien haben, wie generell in den Geisteswissenschaften (vgl. Gräfen/Thielmann 2007: 68, 79) viel größeres Gewicht. Ebenso spielt es für die Wahrnehmung innerhalb der Diskursgemeinschaft keine Rolle, ob man einen Artikel in einer Zeitschrift oder in einem Sammelband veröffentlicht. Die Rolle von Forschungsartikeln, die in Zeitschriften und Sammelbänden mit Review veröffentlicht werden, wird jedoch durch die aktuellen allgemeinen Kriterien der Universitäten für Berufungsverfahren auch in der slawistischen Sprachwissenschaft zunehmend bedeutsamer.

Wir gehen davon aus, dass diese Umstände Auswirkungen auf die inhaltliche und sprachliche Gestaltung von Artikeln in deutscher Sprache haben:

1. Erstens ist es für deutschsprachige SlawistInnen einfacher auf Deutsch zu schreiben, das heißt, die Herstellung eines Artikels braucht weniger Zeit - z. B. entfällt die sprachliche Korrektur;

\footnotetext{
${ }^{2}$ Anzeiger für slavische Philologie, Welt der Slaven, Wiener slavistisches Jahrbuch, Wiener slawistischer Almanach, Zeitschrift für slavische Philologie, Zeitschrift für Slawistik.
} 
2. Zweitens ist die Publikation in einem Sammelband oder einer Zeitschrift, wo im Wesentlichen die HerausgeberInnen über die Annahme und Überarbeitung eines Artikels entscheiden, weniger aufwändig als bei einem formalisierten Peer-review-Verfahren;

3. Drittens ist die Rezeption eines deutschsprachigen Artikels von vornherein auf eine bestimmte Diskursgemeinschaft beschränkt, (diese ist jedoch nicht zwangsläufig kleiner als eine slawischsprachige, man denke etwa an die Slowenistik).

Wir vermuten daher, dass deutschsprachige slawistische Artikel, die in Zeitschriften und Sammelbänden erscheinen, inhaltlich und sprachlich nicht besonders elaboriert sind, bzw. der Grad der Elaboration individuell variiert, da sie für eine spezifische und kleine Diskursgemeinschaft verfasst werden. Daher können bestimmte Informationen implizit und der Inferenz durch die AdressatInnen überlassen bleiben.

Was bedeutet dies nun für die fachliche (und sprachliche) Sozialisation der Studierenden?

\section{$2.2 \quad$ Fragestellung}

Studierende, die ein Sprachstudium wählen, haben gegenwärtig zumeist das Ziel, die gewählte Sprache zu erlernen und darüber hinaus interessante Fakten über die mit dieser Sprache verbundene Kultur und Literatur zu erfahren, und erwarten von den Lehrenden gesichertes Wissen, das ihnen diese qua ihrer Autorität in geeigneter Weise zum Erlernen zur Verfügung stellen. Sie sehen das Studium ,als Rezeption und Reproduktion von vorhandenen Wissensbeständen“ (Sesink 2012: 10). Der Prozess der Wissensproduktion steht nach unserer Erfahrung nicht im Mittelpunkt ihres Interesses und damit auch nicht die „kreative Beteiligung am Prozess der Wissenschaft" (Sesink 2012: 10). Letzteres ist aber Auftrag der Universitäten: „Im gemeinsamen Wirken von Lehrenden und Studierenden wird in einer aufgeklärten Wissensgesellschaft das Streben nach Bildung und Autonomie des Individuums durch Wissenschaft vollzogen" heißt es etwa im österreichischen Universitätsgesetz (UG 2002, §1, www.ris.bka.gv.at/GeltendeFassung.wxe?Abfrage=Bundesnormen \&Gesetzesnummer=20002128\&FassungVom=2015-10-19, [28.12.2015]).

Auf Grund dieses Bildungsauftrages gilt es (auch im Bachelorstudium), neben den aktuell als gesichert geltenden Wissensbeständen, zu vermitteln, dass Wissen ständig hinterfragt und erneuert werden muss und dass es dazu bestimmter Herangehensweisen bedarf. Durch das universitäre Studium sollen Studierende dazu befähigt werden, wissenschaftliche Erkenntnisse von Behauptungen $\mathrm{zu}$ unterscheiden. Dazu müssen sie verstehen, wie akademisches Wissen produziert wird. Aus eben diesem Grund kommt hier die Textsorte des Forschungsartikels ins Spiel.

Zur Vermittlung von deklarativem Wissen sind Handbuchartikel, Grammatiken und Lehrbücher geeignet. Aus diesen Texten lässt sich aber der Forschungsprozess nicht nachvollziehen - das gilt jedenfalls für entsprechende Publikationen der slawistischen Sprachwissenschaft. Forschungsartikel hingegen präsentieren wissenschaftliche Erkenntnisse als Ergebnis einer Problemlösung (vgl. Graefen 1997: 58, Petkova-Kessanlis 2009: 127). Sie sollen eine Forschungslücke füllen. Deswegen müssen sie an den bestehenden wissenschaftlichen Diskurs anknüpfen und ihr Problem darin theoretisch verorten (vgl. Graefen/Thielmann 2007: 72). Und sie müssen zeigen, auf welche Weise, durch welche ISSN 1615-3014 
Methode, sie die Forschungslücke schließen. Insofern sind sie als Mittel zur fachlichen Sozialisation geeignet.

Allerdings werden Forschungsartikel für gleichberechtigte WissenschaftlerInnen geschrieben (Graefen 1997: 82-83), für die Scientific Community - nicht für Studierende. Und sie müssen genau für die jeweilige wissenschaftliche Gemeinschaft relevant sein (im Sinne von Grice), d. h., nicht $\mathrm{zu}$ viel und nicht $\mathrm{zu}$ wenig kommunizieren. Dazu kommt außerdem die Beschränkung des Umfangs, der nur eine bestimmte Ausführlichkeit zulässt - im Gegensatz zur monografischen Darstellung (Graefen/Thielmann 2007: 97). Insofern sind Forschungsartikel didaktisch weniger geeignet, weil sie auf ein spezifisches Vorwissen abheben, das Studierende noch nicht besitzen. Andererseits ermöglichen sie dadurch jedoch, dass bei den Studierenden Fragen entstehen und sie sich dieses Vorwissen aus anderen Quellen aneignen. Der beschränkte Umfang von Forschungsartikeln ist aber auch ein „Vorteil der Kürze“: Sie können rascher rezipiert werden als Monografien und schrecken Studierende nicht bereits auf Grund ihrer Länge ab.

Es stellen sich nun folgende Fragen: Was erwartet die Studierenden, wenn sie erstmals slawistische Forschungsartikel in deutscher Sprache rezipieren? Können Sie durch die Lektüre und Bearbeitung herausfinden, wie slawistische Forschung betrieben wird? Diese Frage wollen wir versuchen, durch eine Analyse der Inhaltsstruktur von zehn sprachwissenschaftlichen Artikeln der Slawistik zu beantworten.

\subsection{Material und Vorgehensweise}

Die Auswahl der Forschungsartikel war an die eingangs erwähnte didaktische Maßnahme gebunden: Die Artikel wurden den Studierenden vorgeschlagen und waren so gewählt, dass sie ohne großen theoretischen Apparat auskommen, daher wurden Bereiche der formalen Grammatik und Semantik ausgeklammert. Sie sollten systemlinguistische Themen jener slawischen Sprachen behandeln, die im Slawistikstudium an der Universität Klagenfurt gewählt werden können (Bosnisch, Kroatisch, Serbisch, Slowenisch, Russisch), und theoretisch und methodisch aktuell sein. Die Studierenden sollten an Hand eines Fragenkatalogs herausfinden, was das Thema des Artikels ist und welche Forschungsfrage verfolgt wird, auf welche Weise, d. h., mit welcher Methode die Autoren und Autorinnen die Fragestellung bearbeiten und wie diese theoretisch eingeordnet wird.

Aus der Literaturliste mit etwa 30 Titeln haben wir zehn Artikel, die zwischen 1992 und 2007 publiziert wurden, für unsere gegenwärtige Analyse ausgewählt. Davon sind acht in Sammelbänden erschienen, zwei in Zeitschriften. Neun der Artikel sind im Rahmen der oben (2.1) beschriebenen alljährlichen Symposien von Slawistenkreisen entstanden. Fünf der Artikel stammen von JungwissenschaftlerInnen (vor oder kurz nach der Promotion), fünf hingegen von etablierten ProfessorInnen. ${ }^{3}$ Alle Artikel sind deskriptiv und stützen sich zumindest zum Teil auf eigene empirische Forschung. Insofern sind sie Instanzen der Textsorte Forschungsartikel.

In unserer Analyse haben wir uns am Fragenkatalog der Studierenden orientiert und

\footnotetext{
${ }^{3}$ Auf Grund der erwähnten geringen Größe der Forschungsgemeinschaft bleiben die AutorInnen anonym.
} 
Textstellen, die den Fragen entsprachen, in den ausgewählten zehn Artikeln identifiziert und unsere Ergebnisse in der Folge mit jenen von Petkova-Kessanlis (2009) und Graefen/Thielmann (2007) verglichen. Da es sich, wie in der Einleitung erwähnt, um eine explorative Studie handelt, kann und sollte diese Analyse präzisiert und vertieft werden. Im vorliegenden Beitrag wollen wir auf lediglich drei Elemente des Fragenkatalogs eingehen: 1) Formulierung der Forschungsfrage, 2) Methode und 3) theoretische Einordnung.

Der Fragenkatalog enthielt dazu folgende Fragen:

1. Was ist die Forschungsfrage, der nachgegangen wird? Oder was ist der Zweck des Artikels? Was will er herausfinden oder darstellen?

2. Wie wird dieser Frage nachgegangen?

3. a. Was sind wichtige Begriffe bzw. wichtige Termini? - b. Auf welche grundlegende Literatur stützt sich der Artikel? Wer hat noch zu diesem speziellen Thema geschrieben? c. Auf welche theoretische Grundlage stützt sich der Artikel?

Frage 1) hebt auf die Formulierung der Forschungsfrage und/oder des Zieles des Artikels ab, d. h., was er darstellen, ausführen, zeigen oder beweisen will. Darunter fällt auch die Darstellung der Forschungslücke und die Formulierung von Hypothesen. Diese inhaltlichen Punkte sind nach Petkova-Kessanlis (2009: 198) im einleitenden Textteil zu finden.

Bei Frage 2) geht es um die Darstellung der Methode (untersuchtes Material, Gewinnung, Vorgehensweise, Bearbeitung, Interpretation), die in naturwissenschaftlichen Artikeln im Mittelteil, meist in einem eigenen Methodenteil, zu finden ist (Graefen/Thielmann 2007: 7478).

Frage 3a) nach wichtigen Begriffen und Termini betrifft $u$. a. die Definition der zentralen Begriffe. Frage 3b) nach der grundlegenden Literatur und anderen AutorInnen, die das Thema behandelt haben, hebt auf Vorarbeiten, Hintergrund und Relevanz für die Forschung ab. Es geht dabei einerseits um die Skizzierung des Standes der Wissenschaft und um den Beitrag früherer Studien. Dies ist nach Petkova-Kessanlis (2009: 205) Teil der Einleitung. Es geht aber auch um die Auseinandersetzung mit den Theorien und Ergebnissen anderer, die, nach dem Befund von Graefen/Thielmann (2007: 80) zu schließen, anlassbezogen im Text auftritt. Frage 3c) spricht alle sprachlichen Realisierungen des theoretischen Hintergrunds an (Stand der Wissenschaft, Hintergründe, Relevanz der Arbeit, Definition von Begriffen, der Klärung des Verhältnisses zu Theorien anderer), die, wieder auf Grundlage von Graefen/Thielmann (2007: 80), in geisteswissenschaftlichen Artikeln entsprechend dem Gang der Argumentation realisiert sein können.

\subsection{Ergebnisse}

\subsubsection{Forschungsfrage}

Betrachten wir zunächst die sprachliche Realisierung der Forschungsfrage, die wie erwähnt in der Regel in der Einleitung thematisiert wird. Die Forschungsfrage wurde in 9 von 10 Artikeln in der Einleitung angegeben. In einem Artikel hingegen wird die Forschungsfrage 
lediglich im Titel folgendermaßen angedeutet: ${ }^{4}$ „Die Bedeutung des Slowenischen für die Toponymie der Tauernregion“ (in unserer Reformulierung: „Welche Bedeutung hat das Slowenische für die Toponymie der Tauernregion?"). Es handelt sich hier um eine etymologisch-onomastische Arbeit einer arrivierten Forscherpersönlichkeit.

Die Forschungsfrage kann in unserem Material als Frage oder als Zielstellung formuliert sein. Diese Formulierung kann mehr oder weniger ausführlich sein. Sehr kurz ist die Darstellung in einem Aufsatz einer JungforscherIn mit dem Titel „Zum Ausdruck von GewißheitUngewißheit im Russischen“":

(1) „Am Beispiel epistemisch motivierter Bewertungen will ich zeigen, dass auch die informationelle Austauschebene beeinflusst werden kann.“ (S. 2)

Dieser Satz findet sich auf der zweiten Druckseite des Artikels (S. 2) als vorletzter Satz der Einleitung, die der theoretischen Einordnung des Themas dient. In (1) wird sowohl implizit die Fragestellung - die Untersuchung epistemisch-modaler Ausdrücke - als auch die Lösung bzw. das Ziel (Petkova-Kessanlis 2009: 198, 215-229) - was gezeigt werden soll, nämlich die Beeinflussung der informationellen Austauschebene - genannt.

In manchen Fällen sind die Forschungsfragen so formuliert, dass nicht gänzlich klar wird, auf welche Weise sie beantwortet werden können, wie in Beispiel (1) oben. Hier wird zwar das Ziel des Artikels formuliert, aber der Zusammenhang zwischen epistemisch motivierten Bewertungen und der (davor explizierten) informationellen Ebene wird nicht explizit hergestellt und erschließt sich auch im weiteren Artikel nur sehr schwierig. Im folgenden Beispiel (2) wird der Fokus der Forschungsfrage aus der gewählten Formulierung nicht deutlich. Wie in (1) wird das zu untersuchende Problem als Ziel oder Zweck des Artikels angegeben oder eher angedeutet. Dass es sich dabei um die eigentliche Fragestellung des Artikels handelt, lässt sich aus der Verwendung des Verbs analysieren ableiten:

(2) „Ich halte es für sinnvoll, Szenen, die in sich geschlossene, überschaubare Einheiten sind, als gebundene Textsorten anzusehen und auf den Aspekt-Tempus (A/T) Gebrauch hin zu analysieren." (S. 1)

Diese Fragestellung wird jedoch weder hier noch in weiterer Folge thematisiert. Im Artikel steht nämlich die Analyse des Aspekt-Tempusgebrauchs im Mittelpunkt und nicht die Szenen aus dramatischen Texten (die lediglich das dafür gewählte Untersuchungsmaterial repräsentieren), wie aus seinem Titel „Zum Aspekt/Tempusgebrauch in russischen dramatischen und umgangssprachlichen Dialogen“ zu erkennen ist.

In anderen Fällen werden tatsächlich Fragen gestellt. Die Forschungsfrage kann aus mehreren Teilfragen bestehen. Im folgenden Beispiel (3) aus dem Aufsatz einer arrivierten ForscherIn mit dem Titel „Anglizismen im Russischen: Gamburgery, bifšteksy und die Vaučerisierung Russlands“ finden sich diese Fragen auf der zweiten Druckseite des Artikels, in der Einleitung. Sie dienen gleichzeitig dazu, einen Ausblick auf die Struktur des Mittelteils zu geben, was durch in Klammern gesetzte Verweise deutlich wird:

(3) „Ich möchte versuchen, Antworten auf folgende Frage zu finden:

\footnotetext{
${ }^{4}$ Petkova-Kessanlis (2009: 130-131) verwendet (in einem etwas anderen Zusammenhang) angeben und andeuten zur Bezeichnung unterschiedlicher Grade der sprachlichen Explizitheit.
} 
- Welche Bereiche der Lexik betrifft die Anglifizierung bzw. Amerikanisierung des Russischen?

- Wie werden die Anglizismen von den SprecherInnen weiter bearbeitet (Kap. 3)?

- Welche anderen Ebenen der Sprache sind betroffen (Kap. 4 und 5)?

- Welche Einstellungen haben die SprecherInnen des Russischen zu den Anglizismen (Kap.

6)?

- Welche Normierungstendenzen gibt es auf diesem Gebiet (Kap. 7)?“

Die Teilfragen können jedoch auch an mehreren Stellen des Textes verteilt auftreten, was Beispiel (4) illustriert. Dabei wird die Forschungsfrage zu Beginn (hier auf der ersten Druckseite des Artikels) in allgemeiner Form gestellt und an geeigneten Stellen (dem Argumentationsgang folgend) durch Reformulierungen oder Präzisierungen wieder aufgenommen. Beispiel (4) entstammt der Arbeit einer JungforscherIn mit dem Titel „Zur Verteilung von Möglichkeit und Notwendigkeit im russischen modalen Infinitiv“:

(4) „Im Folgenden wird es um diese implizite Modalität gehen, um die Frage, ob ein einzelner Satztyp die Bedeutung der Notwendigkeit oder der Möglichkeit realisiert; und dann im Weiteren auch, um welchen Modalitätstyp es geht, bzw. was für ein Redehintergrund im Kratzerschen Sinne (Näheres s. unten) anzusetzen ist.“ (S. 1) „Zu welchem Modalitätstyp aber ist er zu rechnen?“ [der Satz] (S. 4)

„Was für ein Redehintergrund ist jetzt bei diesen Satztypen anzusetzen?“ (S. 5)

„Warum lassen diese Sätze nicht die Interpretation des Verbotes zu?“ (S. 6)

„Bevor ich noch eine abschließende Bemerkung zu der Realisierung der Unnötigkeit bringe, möchte ich ein paar Dinge bezüglich des Status dieses Satztyps anmerken.“ (S. 7)

„Womit hängt die Interpretation als Notwendigkeit oder Möglichkeit aber dann zusammen?“ (S. 10)

Wenn wir diesen Befund mit den Ergebnissen von Petkova-Kessanlis (2009: 198) vergleichen, so zeichnet sich ein ähnliches Bild ab: Auch bei nichtslawistischen deutschsprachigen Artikeln fehlt die Forschungsfrage gelegentlich in der Einleitung (PetkovaKessanlis (2009) spricht allerdings nicht von „Forschungsfrage stellen“ sondern von „etwas als Problem deklarieren“ und von „Ziel ankündigen“" als sprachlichen Handlungen). Der Grad der Explizitheit variiert ebenfalls in Petkova-Kessanlis' Material, ebenso die Elaboration der Forschungsfrage wie auch die Platzierung innerhalb des (Teil-)Textes (vgl. Petkova-Kessanlis 2009: 270-273).

Die Forschungsfrage(n) können also mehr oder weniger explizit im Text erscheinen und entsprechend leicht als solche identifiziert werden. Hinzuzufügen ist, dass nur in vier Artikeln eine Forschungslücke genannt bzw. expliziert wird. Dadurch kann in diesen Fällen der Zweck des Artikels eindeutiger festgemacht werden.

\subsubsection{Methode}

Hinsichtlich der Darstellung der Methode verfügen wir über kein Vergleichsmaterial im geisteswissenschaftlichen Bereich. Graefen/Thielmann (2007: 76-77) stellen fest, dass deutschsprachige naturwissenschaftliche Artikel in der Regel einen Methodenabschnitt im Mittelteil aufweisen, der allerdings im Laufe der Jahre immer kürzer geworden ist. Für geisteswissenschaftliche Artikel wird insgesamt eine größere Variation in diesem Bereich 
beobachtet (Graefen/Thielmann 2007: 97) Wir können daher nur konstatieren, dass in unserem Material die Methode(n) an verschiedenen Stellen im Text und sehr unterschiedlich explizit dargestellt werden. Diese Unterschiede scheinen einer gewissen Logik zu folgen:

Je stärker philologisch die Ausrichtung des Artikels ist, desto weniger wird die Methode thematisiert, z. B. gar nicht in dem bereits genannten etymologisch-onomastischen Artikel und beiläufig über den ganzen Text verteilt bei einer übersetzungswissenschaftlichen Arbeit. Jüngere AutorInnen thematisieren die Methode eher als ältere, aber nicht immer in zweckmäßiger Weise, wie anhand von Beispiel (2) erkennbar ist. Am auffälligsten ist, dass eine solche Thematisierung zumeist anlassbezogen an den jeweils relevanten Stellen im Text erfolgt.

Von den zehn Artikeln stellen zwei die Methode ausführlich, in einem eigenen Abschnitt, dar (Beispiele 5 und 6). Ein Artikel stellt das Material ausführlich dar, nicht aber die Art der Auswertung, diese ist nur aus den Ergebnissen erschließbar. Diese drei Artikel stammen von JungforscherInnen. Sechs Artikel enthalten Hinweise auf die verwendete(n) Methode(n), während der bereits bei der Forschungsfrage hervorgehobene etymologisch-onomastische Artikel einer arrivierten ForscherIn als einziger das Wissen um Methoden weitestgehend voraussetzt. In diesem Artikel kann aus einzelnen Wörtern auf die Methode geschlossen werden z. B.: „,der Name Kals (urkundlich 1197 de Calce...)“, wo das Wort urkundlich auf das Studium von alten Dokumenten und Schriften schließen lässt, ,man vergleiche damit heutige slowenische Siedlungsnamen...“ (vergleiche auf eine vergleichende Methode), „ein Blick ins slowenische Wörterbuch..." (Wörterbuch auf die Auswertung von Wörterbüchern).

Das folgende Beispiel ist dem Artikel „Zum Ausdruck von Gewißheit-Ungewißheit im Russischen“ entnommen, der einen kurzen, methodisch gehaltvollen empirischen Teil (2 Druckseiten im Mittelteil) mit der Benennung „Skalierungsexperiment“" enthält:

(5) „In einem Versuch mit schriftsprachlichem Material wurde die Graduierung (Kategorisierung) von modalen und modalisierten Ausdrücken untersucht. Die schriftsprachliche Präsentation des Materials orientiert auf die Aktivierung von Strukturen des internen Lexikons. Den Probanden wurden in einem computergesteuerten Test Stimuli mit folgender Struktur dargeboten:... z. B. Vidno Ivanova., Verojatno Ivanova ${ }^{5}$. Die Aufgabe bestand darin, den Ausprägungsgrad des Kriteriums ,epistemische Sicherheit“ in den einzelnen Äußerungen zu bewerten.“ (1. S.)

„Die Ergebnisse verweisen auf eine semantische Asymmetrie, die sich bereits beim Erarbeiten der Bezeichnungen zeigte" (2. S.)

Der 23 Seiten lange Artikel „Zur Bezeichnung weiblicher Personen im Russischen. Eine empirische Pilotstudie“ enthält einen elf Seiten langen empirischen Teil, der auch so benannt ist, und eine genaue und ausführliche Darstellung der Methode beinhaltet. Die einzelnen Abschnitte dieses Teiltextes sind folgendermaßen betitelt (in Klammern ist der jeweilige Umfang angegeben):

(6) „Design des Befragungsmaterials“ (4 Seiten)

„Auswertung“ (1,5 Seiten)

„Interpretation der Ergebnisse“ (5 Seiten)

\footnotetext{
${ }^{5}$ Die Beispiele erscheinen im Originaltext in kyrillischer Schrift.
} 
In den anderen Artikeln wird wie bereits erwähnt die Methode an verschiedenen Stellen angedeutet, zumeist aber nicht dargestellt, vgl. die folgenden Beispiele $(7,8)$ aus dem Artikel „Wie gehen die slawischen Sprachen mit Anglizismen um? Am Beispiel des Russischen, Tschechischen und Slowenischen“:

(7) Erst dann kann anhand von Momentaufnahmen aus dem Russischen, Tschechischen und Slowenischen deren Umgang mit Anglizismen exemplarisch beleuchtet werden. (S.1, Einleitung)

Die Methode wird hier durch die lexikalischen Elemente Momentaufnahmen und exemplarisch beleuchtet angedeutet.

Im folgenden Beispiel (8) erklärt die AutorIn, dass das verwendete Material aus einer bereits publizierten Quelle stammt (8a.):

(8) a. Da die Verhältnisse im Tschechischen in Bezug auf englische Entlehnungen für den deutschen Leser in einer umfangreichen Monografie (Warmbrunn 1994) gut dargestellt sind, kann hier auf eine ausführliche Darstellung verzichtet werden. Das im Buch dargestellte Material bietet eine Aufteilung auf Sachbereiche, samt quantitativer Angaben, die ich hier mit geringen Veränderungen übernehme [...]; Beispiele ergänzt durch eigene Feldforschung (S. 2425, Mittelteil)

Die Methode besteht also erstens in der Auswertung und Analyse bestehender Forschungsliteratur. Dies muss aus Sicht der Scientific Community in diesem Kontext nicht ausgeführt werden, ist aber dadurch für die Studierenden unter Umständen nicht als Methode erkennbar. Zweitens wird auf eigene Feldforschung hingewiesen, die in der Folge (8b.) als Fragebogenerhebung spezifiziert wird:

(8) b. Das Material, das ich anhand eines eigenen Fragebogens ${ }^{18}$ überprüft und ergänzt habe, dokumentiert eindrucksvoll, die ,gemäßigte Akzeptanz“ (Warmbrunn), die das Verhältnis der tschechischen Sprache zur Aufnahme von Anglizismen charakterisiert. (S. 26, Mittelteil)

Genaueres dazu finden wir in einer Anmerkung (8c.), was unseres Erachtens als Andeutung der Methode klassifiziert werden kann:

(8) c. ${ }^{18}$ An dieser Stelle möchte ich mich bei allen bisher 46 InformantInnen (31 w, $\left.15 \mathrm{~m}\right)$ bedanken, sowie für das Sammeln von Fragebögen bei M.F. (Prag), A.B. (Brünn), E.L. (Olmütz), O.R. (Königgrätz), S.E. (Graz/Prag). [...] (S. 36, Anmerkungen)

In dem bereits genannten Artikel einer JungforscherIn mit dem Titel „Zur Verteilung von Möglichkeit und Notwendigkeit im russischen modalen Infinitiv“ finden wir Hinweise auf die Methode ebenfalls an verschiedenen Stellen, und zwar zunächst zu Beginn des Textes (durch die lexikalischen Elemente Paraphrasierungsmöglichkeiten und Übersetzung in 9a.) und weiter in Form von Konkretisierungen dieser Herangehensweise (9b.: durch eine ,, in Hinsicht auf... "-Phrase benennen, 9c. Interpretation, manche Informanten):

(9) a. Bei der Bestimmung, um was für eine Modalität es sich handelt, stütze ich mich auf die Paraphrasierungsmöglichkeiten oder [...] auf die deutsche Übersetzung. (S. 1)

b. Im Anschluss an Kratzer (1978) gehe ich nicht von mehreren Müssen aus, die man durch Indexzahlen zu trennen hätte, sondern von einem Müssen, das seine verschiedenen Interpretationen durch verschiedene Redehintergründe erhält. [...] Explizit kann man diese 
Redehintergründe durch eine ,in Hinsicht auf..."-Phrase benennen. (S. 2)

c. Hierzu muss man zwei Dinge anmerken: Zum einen ist [...] die Interpretation des Satzes als Unnötigkeit die naheliegendere, manche Informanten zögern auch bei der anderen Interpretation [...] (S. 4)

Wir haben es in diesem Fall mit einem Beitrag zur Satzsemantik zu tun, also mit einem grammatiktheoretischen Thema. Die geringe Thematisierung der Methode in den meisten Artikeln hat - so unsere Hypothese - unmittelbar mit diesem Faktum zu tun: In der Grammatikforschung gibt es seit der Zeit des Strukturalismus Erkenntnisprozeduren (wie etwa die verschiedenen Discovery Procedures der strukturalistischen Syntax Verschiebeprobe, Tilgungsprobe, Ersatzprobe), deren Darstellung im Kontext eines wissenschaftlichen Artikels für die Scientific Community nicht relevant wäre. $\mathrm{Zu}$ diesen Methoden gehört u. a. auch die in der Generativistik angewandte Introspektion sowie das Urteil des kompetenten Sprechers.

In den Beispielen (9 a.-c.) finden wir beides: die Andeutung der Paraphrasierung als Erkenntnisprozedur zu Beginn (9a.), deren Konkretisierung mit „,in-Hinsicht-auf-Phrasen“ (9b.) und zuletzt die Erwähnung von „Informanten“ (9c.) als kompetenten SprecherInnen. In der slawistischen Grammatikforschung ist es bislang nicht üblich, diese Befragung von InformantInnen zu quantifizieren (es ist allerdings möglich). Es genügt, darauf hinzuweisen, dass man sich nicht auf sein eigenes Urteil verlässt - was in einer fremdsprachlichen Philologie wie der Russistik relevant ist.

Zusammenfassend lässt sich sagen: Über die Methode wird in unserem Material (wenn überhaupt) zumeist anlassbezogen an verschiedenen Stellen im Mittelteil geschrieben. Die Methoden werden ausführlich und in eigenen Abschnitten beschrieben, wenn es sich um die Darstellung eines Experiments handelt, also um eine Herangehensweise der Natur- und Sozialwissenschaften. Handelt es sich jedoch um die für die Grammatik- und Lexikforschung traditionellen geisteswissenschaftlichen Methoden der Auswertung von schriftlichen Quellen, des mehr oder weniger systematischen Sammelns von Belegen für ein Phänomen, der Beobachtung des Sprachgebrauchs und der InformantInnenbefragung, so wird die Methode meist nur angedeutet oder bleibt implizit, d. h., ihre Kenntnis und die Sinnhaftigkeit ihrer Anwendung wird vorausgesetzt.

\subsubsection{Theoretische Einordnung}

Das dritte Element der Inhaltsstruktur wissenschaftlicher Artikel, das wir untersucht haben, ist die theoretische Einordnung des Themas in den Forschungszusammenhang. Hier gilt für unser Material generell: Die untersuchten sprachlichen Phänomene werden nur sehr kurz dargestellt, wenn überhaupt, und es gibt keine Verweise auf Grammatiken oder andere einschlägige Literatur, aus der man darüber mehr erfahren könnte. Lediglich zentrale Begriffe werden erläutert (10a.), andere Termini werden vorausgesetzt, z. B. wird in dem Artikel „Genus und (Ko-)Referenz“ einer JungforscherIn die Abkürzung NP (für Nominalphrase) nicht aufgelöst (10b.):

(10) a. Wenn man über Genus spricht, muss man folgende beiden Aspekte dieser grammatischen Kategorie berücksichtigen: Erstens ist das Genus ein inhärentes Merkmal von 
Substantiven. In dieser Eigenschaft kann es morphologisch am Substantiv signalisiert sein. Im Russischen wird dies durch die weitgehende Übereinstimmung von Genus und Flexionsklasse realisiert: [...]

b. in nicht-referentiellen NPs, wo der Referent unbestimmt ist, und nicht direkt identifiziert werden muss, hat das Genus keinen so hohen semantischen Gehalt, und mangels besserer Möglichkeiten tritt im Deutschen in der Regel das Maskulinum auf.

Theorien anderer werden kaum beschrieben oder diskutiert sondern zumeist in Form von Zitaten für die eigene Argumentation herangezogen, wie im Artikel „Zum Ausdruck von Gewißheit-Ungewißheit im Russischen“:

(11) Der Bewertungsaspekt ist in jeder Kommunikation präsent: Ein Satz (besser: eine Äußerung) drückt „stets nur ... einen in eine Einstellung gefassten Gedanken aus“ (Lang 1981: 95). Oder, in anderer Terminologie: „Sachverhaltsbeschreibung und Sprecheranteil sind komplementär; nur beide zusammen machen einen Satz (eine Äußerung) aus“ (Wildenhahn 1983: 391). Diese Feststellungen lassen sich auf den Bereich der epistemischen Modalität übertragen.

Das bedeutet aber, dass oft kein Bezug auf den Stand der Forschung genommen wird. In sechs von zehn Artikeln fehlt zudem das Aufzeigen einer Forschungslücke (vgl. Petkova-Kessanlis 2009: 242, die die Konstatierung eines Mangels als ein immer wiederkehrendes Merkmal sprachwissenschaftlicher Artikel nennt).

\section{Diskussion und Schlussfolgerungen}

Die Ergebnisse unserer Forschung bestätigen im Großen und Ganzen unsere Hypothese von der Implizitheit und Indirektheit der slawistischen sprachwissenschaftlichen Artikel. Besonders augenfällig sind die wenig explizite Darstellung der Methoden und der Theorie sowie der Forschungslücke.

Aus unserer Sicht hat dies mit verschiedenen sozialen Parametern zu tun, die die Publikationspraxis in der Slawistik bestimmen: Wie bereits oben angedeutet, stehen die Methoden der geisteswissenschaftlich und/oder systemlinguistisch orientierten Sprachwissenschaft außer Zweifel und müssen in einem Artikel nicht thematisiert, begründet oder verteidigt werden. Lediglich die Verwendung ,neuer“, nichttraditioneller Methoden wie etwa die Durchführung eines Experiments bedürfen einer besonderen Darstellung. Eine explizit(er)e Darstellung der Methoden finden wir zudem bei den JungforscherInnen.

Hinzu kommt als weiterer Faktor der Stellenwert der Monografie in der Slawistik. Artikel, wie wir sie analysiert haben, stellen entweder kleinere explorative Forschungsarbeiten vor oder sind Publikationen von Teilergebnissen, aber auch Überlegungen während der Arbeit an einem größeren Forschungsprojekt, das in eine Monografie mündet. Solche größere Studien arbeiten in der Regel mit klar abgegrenzten Textkorpora und beziehen Befragungen ein. Diese werden nach Abschluss der Forschung in einer Monografie im Detail abgehandelt (vgl. dazu auch Graefen 1997: 101; Graefen und Thielmann 2007: 79). Ebenso werden die Forschungsergebnisse eines größeren Projekts in der Regel ausschließlich in Form einer Monografie publiziert und seltener in einzelnen Zeitschriftenaufsätzen (vgl. Gräfen 1997: 58). Der hohe Stellenwert der Monografie ist auch einer der Gründe dafür, dass die theoretische Einordnung in Forschungsartikeln wenig expliziert wird. Diese Einordnung erfolgt ebenso 
wie die Darstellung der Methode, wie auch Graefen und Thielmann (2007: 79) feststellen, ausführlich in Monografien. Dies hat nach Graefen und Thielmann (2007: 79) mit der Besonderheit der Geisteswissenschaften zu tun, den Gegenstand ihrer Forschung immer wieder neu (er)klären zu müssen.

Die Publikationssprache Deutsch könnte ein weiterer Grund für die geringe Elaboration von Methode und theoretischem Hintergrund sowie der Darstellung der Forschungslücke in unseren Artikeln sein: Wichtigere slawistische Artikel werden in anderen Sprachen veröffentlicht, zumeist in einer slawischen Sprache. Zudem handelt es sich bei der deutschsprachigen slawistischen Sprachwissenschaft um eine relativ kleine Forschungs- und Diskursgemeinschaft: Der Personenkreis ist überschaubar und man kennt die AkteurInnen in der Regel sogar persönlich. All dies führt unseres Erachtens zu einer erhöhten Toleranz gegenüber wenig expliziten Texten oder Textstellen.

Für die Sozialisation von Studierenden in der deutschen Wissenschaftssprache ebenso wie im wissenschaftlichen Arbeiten ist dies allerdings problematisch. Denn die slawistischen Forschungsergebnisse werden in den Artikeln als Ergebnisse des Denkprozesses der AutorInnen dargestellt, bzw. als eine Mischung von Rezeption von Literatur, empirischer Forschung und eigenen Schlüssen. Der Weg dorthin, also die Methode, der Sinn, die Schließung einer Forschungslücke und die Einbettung in den wissenschaftlichen Diskurs, werden jedoch nicht genügend deutlich.

Daraus ziehen wir zweierlei Schlüsse: Erstens eignen sich slawistische Artikel in deutscher Sprache, von einzelnen Ausnahmen abgesehen, nicht besonders gut, um Studierende mit dem wissenschaftlichen Erkenntnisprozess bekannt zu machen. Zweitens eignen sich die Artikel jedoch auch nicht als Vorbild für die akademischen Arbeiten, die Studierende im Studium verfassen sollen.

\section{Literaturverzeichnis}

Ammon, Ulrich (1998): Ist Deutsch noch internationale Wissenschaftssprache? Englisch auch für die Lehre an den deutschsprachigen Hochschulen. Berlin: De Gruyter.

Clyne, Michael (1987): "Cultural Differences in the Organization of Academic Texts, English and German". Journal of Pragmatics 11: 211-247.

Clyne, Michael (1991): „Zu kulturellen Unterschieden in der Produktion und Wahrnehmung englischer und deutscher wissenschaftlicher Texte“. Informationen Deutsch als Fremdsprache 18: 376-383.

Doleschal, Ursula (2007): "Implicitness in Academic Texts. A Case Study of Slovene and Russian Texts". In: Doleschal, Ursula/Gruber, Helmut (eds.): Academic Writing in Languages other than English. Frankfurt a. M., Lang: 59-79.

Graefen, Gabriele (1997): Der wissenschaftliche Artikel - Textart und Textorganisation. Frankfurt a. M.: Lang.

Graefen, Gabriele/Thielmann, Winfried (2007): „Der wissenschaftliche Artikel“. In: Auer, Peter/Baßler, Harald (eds.): Reden und Schreiben in der Wissenschaft. Frankfurt a. M., Lang: 67-98.

Petkova-Kessanlis, Mikaela (2009): Musterhaftigkeit und Varianz in linguistischen ISSN 1615-3014 
Zeitschriftenaufsätzen. Frankfurt a. M.: Lang.

Pöckl, Wolfgang (1995): „Nationalstile in Fachtexten? Vom Tabu- zum Modethema“. Fachsprache 17, 3/4: 98-107.

Sesink, Werner (2012): Einführung in das wissenschaftliche Arbeiten: inklusive E-Learning, Web-Recherche, digitale Präsentation u.a. 9., aktualisierte Aufl. München: Oldenbourg.

Swales, John (1990): Genre Analysis. English in Academic and Research Settings. Cambridge: Cambridge University Press.

Zeil, Wilhelm (1994): Slawistik in Deutschland: Forschungen und Informationen über die Sprachen, Literaturen und Volkskulturen slawischer Völker bis 1945. Köln/Wien: Böhlau. 